\title{
Análisis del método ordinal de decisión multicriterio del Veto
}

\author{
Analysis of the multi-criteria decision-making Veto method \\ Ángel Santiago Fernández Bou ${ }^{1} \quad$ João Carlos Soares de Mello ${ }^{1}$ \\ Recibido 14 de agosto de 2013, aceptado 13 de enero de 2015 \\ Received: August 14, 2013 Accepted: January 13, 2015
}

\begin{abstract}
RESUMEN
No existe ningún método ordinal de apoyo a la decisión que sea perfecto, es decir, un método ordinal no puede matemáticamente cumplir los cinco axiomas de Arrow (independencia de las alternativas irrelevantes, transitividad, universalidad, unanimidad de Pareto y orden total). Por ello, los investigadores han centrado sus iniciativas en buscar métodos que reduzcan la influencia de las alternativas irrelevantes sin incurrir en intransitividad. En 2012 Morais y Teixeira [1] presentaron en la revista Omega un método de decisión multicriterio ordinal, aquí denominado del Veto. Este método consiste en desconsiderar, para el cálculo, los dos cuartiles intermedios de la matriz de alternativas ordenadas por cada tomador de decisión; así, el cuartil superior representará la fuerza de cada alternativa, y el inferior la debilidad, siendo elegida la alternativa con mayor índice de fuerza menos debilidad. Este artículo analizó el método del Veto para la dependencia de alternativas irrelevantes. Para ello se usó como referencia el método de Copeland, reforzado con otros métodos y ejemplos. Las conclusiones indican que el método del Veto es más sensible a alternativas irrelevantes que el de Copeland. El veto de los cuartiles intermedios, postulado para reducir la dependencia de alternativas irrelevantes, no la reduce (puede, incluso, eliminar información esencial para una ordenación justa). Por su estructura, el método del Veto permite la manipulación de las alternativas para influir sobre el resultado, siendo posible forjar una decisión para quien decide qué alternativas serán votadas. Por tanto, el método de Copeland debe preferirse al método del Veto.
\end{abstract}

Palabras clave: Decisión en grupo, análisis multicriterio, ranking, método del Veto, método de Copeland, sensibilidad a alternativas irrelevantes.

\section{ABSTRACT}

There is no perfect ordinal decision-making method. This means, one ordinal method cannot meet the five Arrow Axioms (independence of irrelevant alternatives, transitivity, universality, Pareto unanimity and total order). For this reason, researchers have been focusing their efforts on finding methods that reduce the influence of irrelevant alternatives without incurring intransitivity. In 2012, Morais and Teixeira published at Omega journal the Veto method, which is an ordinal multi-criteria decision method. It consists in removing the intermediate quartiles of the alternatives matrix after it is ranked by the decision makers in order to calculate the chosen alternative; thus, the upper quartile is used to calculate the strength of each alternative and the lower quartile corresponds to the weakness. The alternative chosen is the one with the greatest value of strength minus weakness. This article analyzes the Veto method for the dependence of irrelevant alternatives. To achieve this, the Copeland method was used as reference, reinforced with other methods and examples. The result was that the Veto method is more sensitive to irrelevant alternatives than the Copeland method. The veto of the intermediate quartiles, postulated to reduce the dependence on the irrelevant alternatives, does not reduce it and it can remove essential information to get a fair ranking. Because of its structure, the Veto method allows the manipulation of

1 Escuela de Ingeniería. Universidade Federal Fluminense. Rua Passo da Pátria 156, 22210-240. Niterói. Brasil. E-mail: asfernandezbou@id.uff.br; jcsmello@pq.cnpq.br 
the alternatives to influence the result, making possible for the one who selects the available alternatives to build a decision. Therefore, the Copeland method must be preferred to Veto method.

Keywords: Group decision, multi-criteria analysis, ranking, Veto method, Copeland method, sensitivity to irrelevant alternatives.

\section{INTRODUCCIÓN}

Tomar decisiones correctas es la base de una buena planificación estratégica. No obstante, esta puede ser una ardua tarea [2], especialmente cuando las decisiones deben ser tomadas en grupo.

La literatura [3] distingue dos procedimientos para la toma de decisiones. Un procedimiento sería la agregación de juicios individuales, donde el grupo decide como una unidad; el otro sería la agregación de prioridades individuales, en que cada componente del grupo defiende su propia postura. Esta dicotomía puede entenderse con dos ejemplos, donde el primer procedimiento estaría representado por la junta directiva de una empresa, en la que los directores deben llegar a un consenso con el objetivo de maximizar el lucro, y el segundo procedimiento por un debate entre sindicatos, empresas y gobierno para legislar sobre derechos de los trabajadores. Se puede entender que, en el caso de un debate plural donde cada sector representado en el grupo tiene un objetivo diferente, cada uno de los representantes intentará que las decisiones tomadas por el grupo favorezcan al máximo a las personas a las que representa, a pesar de que eso signifique perjudicar a los otros.

La estructura de cualquier método ordinal estándar puede ser descompuesta en etapas [4], a saber: 1. Identificación de los tomadores de decisión, 2. Definición de las alternativas a ser votadas, 3. Definición de los criterios relevantes, 4. Análisis de las alternativas en función de los criterios, 5. Determinación de la relevancia de cada criterio, 6. Ranking de las alternativas por los tomadores de decisión, 7 Análisis de sensibilidad, 8. Recomendaciones y 9. Implementación de las acciones decididas.

Los métodos ordinales se consideran intuitivos y con pocas exigencias, especialmente para los tomadores de decisión, que solo tienen que ordenar las alternativas de acuerdo con sus preferencias [5]. Muchos métodos ordinales se han formulado para intentar establecer el mejor camino para tomar decisiones sujetas a múltiples criterios, pero no existe un método perfecto.

A mediados del siglo XX, Arrow [6-7] formuló los cinco axiomas que sistematizan las condiciones necesarias para que un método no produzca paradojas y sea justo. Esos axiomas son [8]:

- Independencia de las alternativas irrelevantes: dado un par de alternativas $x$ e $y$ dentro de un conjunto mayor de alternativas posibles, la preferencia por $x$ o por $y$ debe depender únicamente de cómo los tomadores de decisión ordenan $x$ respecto de $y$, y no de cómo ordenan las otras alternativas del conjunto.

- Transitividad: si A es preferible a B y B es preferible a $\mathrm{C}$, entonces $\mathrm{A}$ debe ser preferible a $\mathrm{C}$.

- Universalidad: el método debe funcionar respetando todos los axiomas para cualquier conjunto de decisiones de los tomadores de decisión.

- Unanimidad de Pareto: si existen dos alternativas para las que todos los tomadores de decisión prefieran la primera a la segunda, la primera debe quedar posicionada por encima de la otra en la ordenación final.

- Orden total: dado un par de alternativas $x$ e $y$ dentro de un conjunto mayor de alternativas posibles, $x$ tiene que ser mejor, peor o igual que $y$.

Arrow demostró posteriormente que no existe la posibilidad de crear un método que cumpla los cinco axiomas. Por tanto, a pesar de tener carácter científico, esta limitación introduce subjetividad, ya que el método ordinal debe ser seleccionado para cada situación y en función de las características de cada caso [4]. 
El denominado Apoyo a las Decisiones Multicriterio fue constituido formalmente como una rama de la Investigación Operativa durante los años setenta. No obstante, los primordios de esta metodología se dieron durante la Revolución Francesa, a finales del siglo XVIII [9], cuando aparecieron dos importantes métodos ordinales, Borda e Condorcet, que dieron inicio a las Escuelas Americana y Francesa, respectivamente. Ambos métodos ya habían sido vislumbrados por el erudito mallorquín Ramón Llull en el siglo XIII, si bien de forma similar y con notación matemática, no exactamente igual. En cualquier caso, los manuscritos donde aparecía el desarrollo de los sistemas de votación de Llull ("Ars notandi", "Ars eleccionis" y "Alia ars eleccionis") fueron publicados recientemente [10], no deslegitimando, por lo tanto, la originalidad de los matemáticos franceses.

El método de Borda [11], también conocido como Recuento Borda, fue creado en el siglo XVIII por Jean-Charles de Borda, ilustre caballero francés, que se destacó, no solo en matemática, como también en física, astronomía, ingeniería y náutica. Según este método, cada tomador de decisión debe ordenar las alternativas (candidatos) acorde a su preferencia individual; así, se crea entre todos los tomadores de decisión una matriz donde las columnas representan las preferencias de cada tomador de decisión y las filas las alternativas ordenadas, siendo más preferidas cuanto más alto se encuentren. Después se asigna 1 punto a la primera fila de alternativas (las favoritas de cada tomador de decisión), 2 puntos a la segunda, 3 puntos a la tercera, y así sucesivamente hasta dar la mayor puntuación posible a las alternativas situadas en la última fila (las menos preferidas por cada tomador de decisión). Vencerá la alternativa que acumule menos puntos. Esta metodología considera todas las preferencias de cada tomador de decisión, no solo la primera; de este modo, la alternativa vencedora puede ser diferente a la que más veces quedó en primer lugar. El método de Borda tiene la ventaja de ser intuitivo y simple, siendo incluso usadas algunas de sus variantes en competiciones deportivas, como la Fórmula 1 [9]. No obstante, tiene una debilidad importante, pues no respeta el axioma de Arrow sobre la independencia de las alternativas irrelevantes [6]. Eso significa que la posición de dos alternativas depende (no es independiente) de su ranking respecto de las alternativas irrelevantes. Esta debilidad hace que el resultado dependa directamente del conjunto de alternativas analizadas y permite la posibilidad de manipulación deshonesta de la votación [12].

El método de Condorcet [13] fue creado pocos años después del de Borda, también en el siglo XVIII, por Marie Jean Antoine Nicolas Caritat, el Marquis de Condorcet. Este método está basado en relaciones de superación entre pares de alternativas, que son representadas con un grafo donde aparece la relación entre dominante y dominada, cuando esa relación existe [8]. Si existe una única alternativa dominante, esa será la elegida [12]. Este método tiene como principal ventaja la independencia de las alternativas irrelevantes, es decir, el resultado de la votación no depende del grupo de alternativas seleccionadas. Sin embargo, el método de Condorcet no siempre consigue dar una ordenación completa, porque no respeta en todos los casos el axioma de Arrow de transitividad, ya que puede llevar a situaciones de intransitividad del tipo "A domina a B, B domina a C y C domina a A". Esta situación se denomina paradoja de Condorcet y hace imposible la ordenación de las alternativas. Tampoco cumple el axioma de universalidad, puesto que no funciona para cualquier conjunto de preferencias de los tomadores de decisión.

Cuando no hay intransitividad en la votación, el método de Condorcet es preferible al método de Borda [14].

El método ordinal más referenciado en la literatura, además del recuento Borda y el método de Condorcet, es el método de Copeland [12]. El método de Copeland [15] deriva del método de Condorcet y usa la misma matriz de relaciones entre pares de alternativas. El cálculo realizado es victorias menos derrotas; es decir, se suma el número de veces que cada alternativa venció a otras y el número de veces que perdió, y la diferencia es el resultado. La alternativa escogida será aquella cuyo resultado sea mayor. El método de Copeland tiene el mismo resultado que el método de Condorcet cuando no existen ciclos de intransitividad, pero cuando existe, a diferencia de este, Copeland es capaz de ordenar las alternativas. Es un poco más complejo que el método de Borda, pero tiene la ventaja sobre este de reducir (aunque sin eliminar en todos los casos) la influencia de las alternativas irrelevantes [16]. 
Así, se puede decir que el método de Copeland aúna la filosofía de los métodos de Borda y de Condorcet y, aunque no es un método perfecto, es en todos los casos igual o mejor que estos dos. De hecho, de acuerdo con Arrow, no existe un método ordinal perfecto, y los esfuerzos realizados por los diferentes investigadores y matemáticos se basan en intentar reducir la dependencia de las alternativas irrelevantes sin caer en intransitividades.

Otro método bien conocido es el lexicográfico, que sí que satisface los axiomas de Arrow. En él, las alternativas son ordenadas en función del criterio más importante. En caso de que haya un empate entre alternativas, este se resuelve considerando la ordenación del segundo criterio en importancia [17]. Por ejemplo, este método podría utilizarse para obtener la clasificación de los países participantes en los Juegos Olímpicos de acuerdo con el número de medallas $[18,19]$ : el criterio principal sería el número de medallas de oro y, en caso de empate, de plata, de bronce, cuartos puestos, etcétera. No obstante, como el método lexicográfico es dictatorial, se requeriría que los tomadores de decisión definiesen previamente qué criterio se comportaría como "dictador", o sea, cuál sería el criterio más importante. Por este motivo no se utilizaron los resultados del método lexicográfico como referencia en este trabajo.

Este artículo analizó el método propuesto por Morais y Teixeira [1], denominado método del Veto, y especialmente su sensibilidad a las alternativas irrelevantes. Para realizar la evaluación del método, se usó como referencia el método de Copeland, por considerar que reduce la dependencia de las alternativas irrelevantes.

\section{EL MÉTODO DEL VETO}

El método del Veto fue creado por Danielle C. Morais y Adiel Teixeira de Almeida, de la Universidad Federal de Pernambuco [1]. En él, los autores pretenden trazar un método que sea aplicable a las decisiones relativas a cuencas hidrográficas, donde los tomadores de decisión tienen intereses claramente enfrentados. Es un método ordinal de decisión multicriterio por medio de ranking de las alternativas, cuyo objetivo es elegir la mejor alternativa de entre las analizadas, de manera que pueda ser considerada apropiada para resolver el problema acorde al punto de vista de todos.
Dado un determinado problema que tiene que ser resuelto adoptando una única medida (alternativa), son considerados $m$ tomadores de decisión y $n$ alternativas $\left(k=\left\{T D_{1}, T D_{2}, \ldots, T D_{m}\right\}\right.$ e $i=\{1,2, \ldots$, $n\})$. Cada tomador de decisión ordena las alternativas colocándolas en orden decreciente desde la favorita hasta la menos preferida. Se parte de la base de que las preferencias de cada tomador de decisión son transitivas, que todos los tomadores de decisión tienen el mismo tratamiento y que una preferencia colectiva es la agregación de varias preferencias individuales.

Una vez ordenadas las alternativas, la matriz resultante se divide en cuartiles de manera que el cuartil superior contendrá el $25 \%$ de las alternativas preferidas por cada tomador de decisión, y el cuartil inferior el $25 \%$ de las alternativas menos preferidas. En una segunda fase, denominada VETO, los dos cuartiles intermedios son eliminados, siendo los cálculos realizados únicamente con las alternativas de los cuartiles extremos. En la tercera fase, el cuartil superior se utilizará para calcular la FUERZA de las alternativas (ecuación (1)), y el inferior para calcular la DEBILIDAD (ecuación (2)). En el caso en que el número de alternativas dentro del cuartil no sea un número entero, se redondeará de forma tradicional, es decir, entre cero y cinco décimas, se redondeará hacia abajo y para cinco décimas o más, hacia arriba. Así, se atribuye un valor correspondiente a cada posición dentro del cuartil, de manera que las alternativas situadas en los extremos tienen más peso que las alternativas más próximas a los cuartiles intermedios.

Matemáticamente:

FUERZA de la alternativa $i$

$$
\begin{gathered}
F_{i}=\sum_{k=1}^{m} \sum_{j=1}^{x}(x-j+1) q_{i j}^{k} \\
\forall i, k \quad \forall j=1,2, \ldots, x
\end{gathered}
$$

DEBILIDAD de la alternativa $i$

Donde

$$
\begin{array}{r}
f_{i}=\sum_{k=1}^{m} \sum_{j=y}^{n}(j-y+1) q_{i j}^{k} \\
\forall i, k \quad \forall j=\mathrm{y}, \ldots, n
\end{array}
$$

$i$ es cada alternativa;

$j$ es la posición de la alternativa $i$ para cada tomador de decisión; 
$k$ es cada tomador de decisión;

$m$ es el número total de tomadores de decisión;

$n$ es el número total de alternativas;

$x$ es la posición de la última alternativa dentro del cuartil superior;

$y$ es la posición de la primera alternativa dentro del cuartil inferior;

$q_{i j}^{k} \begin{cases}1 & \begin{array}{l}\text { si la alternativa } i \text { está en la posición } j \\ \text { para el tomador de decisión } k\end{array} \\ 0 & \text { en cualquier otro caso }\end{cases}$

Posteriormente, se calculará la intensidad de la fuerza de cada alternativa, $\alpha_{i}$, que es la diferencia entre la fuerza y la debilidad (ecuación (3)).

Matemáticamente:

Índice $\alpha$

$$
\alpha_{i}=F_{i-} f_{i}
$$

La alternativa elegida será aquella con el mayor índice $\alpha$.

En la Figura 1 se presenta el esquema del método del Veto.

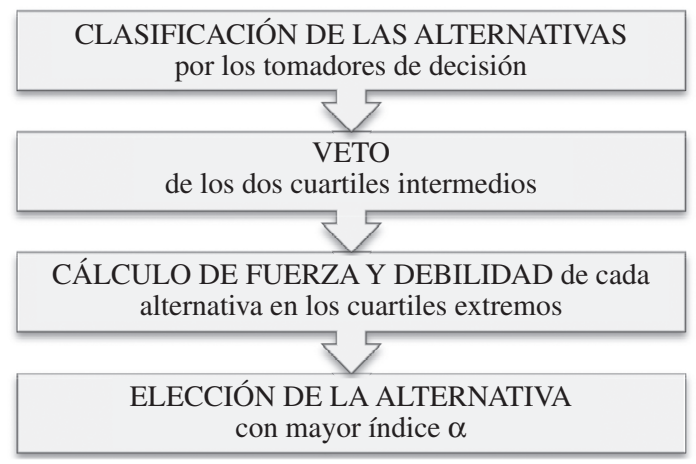

Figura 1. Esquema del método del Veto.

El artículo original incluye el denominado coeficiente $\beta$, que mide la intensidad del desacuerdo del grupo con una determinada alternativa. Así, si la debilidad de una alternativa es $\beta$ veces o más superior a la fuerza de la misma, esa alternativa será descartada (ecuación (4)). El valor de este coeficiente $\beta$ debe ser establecido por los tomadores de decisión asesorados por un consultor.
Matemáticamente:

Coeficiente $\beta$

$$
\text { Si } f_{i}^{3} \beta \cdot F_{i} \rightarrow \mathrm{A}_{i} \text { es descartada }
$$

Es decir, si el cociente $\frac{f_{i}}{F_{i}}$ es suficientemente alto (igual o mayor que el coeficiente $\beta$ ), la alternativa $i$ será descartada.

\section{APLICACIÓN DEL MÉTODO DEL VETO}

Morais y Teixeira aplicaron este modelo dentro de un comité de gestión de una cuenca hidrográfica, para ser capaces de definir una alternativa entre un abanico de posibilidades que fuese la más apropiada para representar al grupo afectado por la decisión. El objetivo era escoger una única alternativa entre todas las presentadas de manera que se controlase satisfactoriamente la degradación ambiental que se da en aquel lugar.

La cuenca hidrográfica objeto de estudio en su artículo fue la del Rio Jaboatão, en el estado de Pernambuco, región nordeste de Brasil. Para ello, en su artículo se explica el marco en que este tipo de gestión se da; en Brasil, las decisiones que afectan la gestión de cuencas hidrográficas están descentralizadas. De esta forma, los comités de cuencas hidrográficas son los responsables de reunir a las partes afectadas, de analizar la situación y, en consenso con las partes, decidir cuál es la mejor solución para resolver cada problema.

En el artículo, los autores estructuraron el proceso conforme el siguiente procedimiento:

(1) Caracterización de la cuenca hidrográfica estudiada.

(2) Identificación de los tomadores de decisión.

(3) Selección de las alternativas.

(4) Ordenación de las alternativas (Ranking).

(5) Decisión del grupo.

La caracterización de la cuenca hidrográfica consiste en un estudio de base para poder adaptar el método del Veto de la mejor manera posible. Un consultor analizó la cuenca para determinar quiénes eran los agentes que están afectados por el proceso, y así definir quiénes serían los tomadores de decisión. 
Como el método se aplicó para identificar la mejor alternativa para combatir la degradación de la cuenca, el consultor investigó las condiciones medioambientales para ser capaz de proponer las alternativas que serían ordenadas por los tomadores de decisión acorde a sus preferencias individuales. Los tomadores de decisión que intervinieron en el proceso fueron representando a las partes interesadas en los recursos hídricos (stakeholders), las Industrias, las Agroindustrias y las Compañías de tratamiento y abastecimiento de agua; representando al sector público, el gobierno Federal, del Estado y Municipal; y representando a la sociedad, las Universidades y asociaciones civiles. Los tomadores de decisión son presentados en la Tabla 1.

Las alternativas seleccionadas por el consultor fueron un total de 12 (Tabla 2). Es decir, cada uno de los cuartiles de la matriz de alternativas ordenadas (Tabla 3 ) quedó compuesto por tres alternativas ( $25 \%$ de 12). Con esta matriz de alternativas ordenadas se realizó el veto, retirando los dos cuartiles intermedios, como se muestra en la Tabla 4.

Los cálculos realizados para determinar la fuerza de las alternativas fueron:

- Tres puntos para las colocadas en la primera posición para cada tomador de decisión: A5, A2, A5, A9, A9.

- Dos puntos para las colocadas en la segunda posición: A2, A5, A4, A6, A4.

- Un punto para las colocadas en la tercera posición: A10, A6, A3, A4, A3.

Y para la debilidad de las alternativas:

- Tres puntos para las colocadas en la duodécima posición para cada tomador de decisión: A1, A1, A1, A1, A11.

Tabla 1. Tomadores de decisión.

\begin{tabular}{|l|l|c|}
\hline \multicolumn{1}{|c|}{ Representación } & \multicolumn{1}{|c|}{ Sector/ entidad } & $\mathbf{N}^{\mathbf{0}}$ \\
\hline \multirow{3}{*}{ Stakeholders } & Industrias & 1 \\
\cline { 2 - 3 } & Agroindustrias & 1 \\
\cline { 2 - 3 } & Compañías de tratamiento y abastecimiento de agua & 1 \\
\hline Sector público & Gobierno Federal, Estadual y Municipal & 1 \\
\hline Sociedad civil & Universidades o asociaciones civiles & 1 \\
\hline
\end{tabular}

Tabla 2. Alternativas.

\begin{tabular}{|c|l|}
\hline Código & \multicolumn{1}{|c|}{ Descripción } \\
\hline A1 & $\begin{array}{l}\text { Tratamiento del sistema de alcantarillado secundario en Jaboatão dos Guararapes, que requiere que } \\
\text { los residuos industriales sean tratados previamente de acuerdo con las normas establecidas }\end{array}$ \\
\hline A2 & $\begin{array}{l}\text { Campañas educativas en las poblaciones que se encuentran dentro de la cuenca hidrográfica (excepto } \\
\text { Recife). }\end{array}$ \\
\hline A3 & $\begin{array}{l}\text { Una campaña con la industria para minimizar la cantidad de agua utilizada en la producción de } \\
\text { procesos que ofrezca incentivos económicos para las industrias que muestren resultados positivos }\end{array}$ \\
\hline A4 & $\begin{array}{l}\text { Mantenimiento de las instalaciones industriales para prevenir que el agua usada para refrigeración } \\
\text { sea contaminada por residuos provenientes de procesos industriales. }\end{array}$ \\
\hline A5 & $\begin{array}{l}\text { Instituir políticas para controlar el desarrollo de nuevas compañías y/o la expansión de las actuales } \\
\text { para evitar empeoramiento causado por la contaminación industrial. }\end{array}$ \\
\hline A6 & $\begin{array}{l}\text { Desarrollar un plan de agricultura sostenible específico para los productores de Vitória de Santo } \\
\text { Antão que esté enfocado a la conservación del suelo y del agua dentro de la cuenca del río Jaboatão. }\end{array}$ \\
\hline A7 & Recuperación de la vegetación nativa a lo largo de las márgenes del río Jaboatão. \\
\hline A8 & Mejorar la colecta de residuos a lo largo del río, así como pautar la recogida periódica de basura. \\
\hline A9 & Recuperación del ecosistema acuático. \\
\hline A10 & $\begin{array}{l}\text { Tratamiento de los puntos de erosión para favorecer la reducción de los procesos de sedimentación } \\
\text { que pueden colmatar los ríos y la red de drenaje de lluvias. }\end{array}$ \\
\hline A11 & Recuperación de la biodiversidad de la fauna \\
\hline A12 & Desarrollo de turismo sostenible con actividades a lo largo del río Jaboatão. \\
\hline
\end{tabular}


Tabla 3. Alternativas ordenadas por cada tomador de decisión.

\begin{tabular}{|c|c|c|c|c|c|}
\hline Ranking & TD1 & TD2 & TD3 & TD4 & TD5 \\
\hline $\mathbf{1}^{\mathbf{0}}$ & A5 & A2 & A5 & A9 & A9 \\
\hline $\mathbf{2}^{\mathbf{0}}$ & A2 & A5 & A4 & A6 & A4 \\
\hline $\mathbf{3}^{\mathbf{0}}$ & A10 & A6 & A3 & A4 & A3 \\
\hline $\mathbf{4}^{\mathbf{0}}$ & A4 & A4 & A2 & A2 & A6 \\
\hline $\mathbf{5}^{\mathbf{0}}$ & A6 & A9 & A10 & A3 & A12 \\
\hline $\mathbf{6}^{\mathbf{0}}$ & A3 & A10 & A9 & A11 & A10 \\
\hline $\mathbf{7}^{\mathbf{0}}$ & A9 & A3 & A6 & A7 & A8 \\
\hline $\mathbf{8}^{\mathbf{0}}$ & A7 & A7 & A7 & A8 & A2 \\
\hline $\mathbf{9}^{\mathbf{0}}$ & A11 & A12 & A11 & A10 & A5 \\
\hline $\mathbf{1 0}^{\mathbf{o}}$ & A12 & A8 & A8 & A5 & A1 \\
\hline $\mathbf{1 1}^{\mathbf{0}}$ & A8 & A11 & A12 & A12 & A7 \\
\hline $\mathbf{1 2}^{\mathbf{0}}$ & A1 & A1 & A1 & A1 & A11 \\
\hline
\end{tabular}

Tabla 4. Matriz de alternativas ordenadas después de aplicar el veto.

\begin{tabular}{|c|c|c|c|c|c|c|}
\hline Ranking & & TD1 & TD2 & TD3 & TD4 & TD5 \\
\hline $1^{\circ}$ & \multirow{3}{*}{ 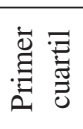 } & A5 & A2 & A5 & A9 & A9 \\
\hline $2^{\mathbf{o}}$ & & A2 & A5 & A4 & A6 & A4 \\
\hline $3^{\circ}$ & & A10 & A6 & A3 & A4 & A3 \\
\hline $10^{\circ}$ & \multirow{3}{*}{ 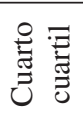 } & A12 & A8 & A8 & A5 & A1 \\
\hline $11^{\circ}$ & & A8 & A11 & A12 & A12 & A7 \\
\hline $12^{\circ}$ & & $\mathrm{A} 1$ & A1 & A1 & A1 & A11 \\
\hline
\end{tabular}

- Dos puntos para las colocadas en la undécima posición: A8, A11, A12, A12, A7.

- Un punto para las colocadas en la décima posición: A12, A8, A8, A5, A1.

La puntuación de las alternativas y el valor de índice $\alpha$ son presentados en la Tabla 5 .
Así, el ranking final de las alternativas, acorde al método del Veto, tiene los resultados presentados en la Tabla 6. La alternativa seleccionada aplicando el método del Veto es la A5.

Tabla 6. Resultado de la aplicación del método del Veto con la ordenación final de las alternativas.

\begin{tabular}{|c|c|c|c|c|c|}
\hline \multicolumn{2}{|c|}{ Alternativa } & Puntos & \multicolumn{2}{|c|}{ Alternativa } & Puntos \\
\hline $1^{a}$ & A5 & 7 & $7^{a}$ & A10 & 1 \\
\hline $2^{a}$ & A9 & 6 & $8^{a}$ & A7 & -2 \\
\hline $3^{\mathrm{a}}$ & A2 & 5 & $9^{\mathrm{a}}$ & A8 & -4 \\
\hline $4^{a}$ & A4 & 5 & $10^{\mathrm{a}}$ & A11 & -5 \\
\hline $5^{\mathrm{a}}$ & A6 & 3 & $11^{\mathrm{a}}$ & A12 & -5 \\
\hline $6^{\mathrm{a}}$ & A3 & 2 & $12^{\mathrm{a}}$ & A1 & -13 \\
\hline
\end{tabular}

\section{ANÁLISIS DEL MÉTODO DEL VETO}

El presente análisis pretende mostrar que el método del Veto es altamente dependiente de las alternativas irrelevantes, y que permite la manipulación de los resultados basada en la elección de las alternativas. Como referencia principal se usará el método de Copeland, que consiste en la comparación entre pares de alternativas; al número de veces que una alternativa superó a otra para todos los tomadores de decisión se resta el número de veces que esa alternativa perdió de otra. Vence la alternativa con más puntos. La matriz de relaciones de dominancia entre las alternativas para todos los tomadores de decisión se presenta en la Tabla 7.

El resultado del método de Copeland se muestra en la Tabla 8.

Tabla 5. Resultados parciales y final aplicando el método del Veto.

\begin{tabular}{|c|c|c|c|c|c|c|c|c|c|}
\hline \multirow{2}{*}{ Alternativa } & \multicolumn{4}{|c|}{ FUERZA } & \multicolumn{4}{|c|}{ DEBILIDAD } & \multirow{2}{*}{$\alpha$} \\
\hline & $\mathbf{1}^{\mathrm{a}}$ & $2^{\mathrm{a}}$ & $3^{\mathrm{a}}$ & Subtotal & $10^{\mathrm{a}}$ & $11^{\mathrm{a}}$ & $12^{\mathrm{a}}$ & Subtotal & \\
\hline A1 & - & - & - & 0 & 1 & - & 4 & 13 & -13 \\
\hline A2 & 1 & 1 & - & 5 & - & - & - & 0 & 5 \\
\hline A3 & - & - & 2 & 2 & - & - & - & 0 & 2 \\
\hline A4 & - & 2 & 1 & 5 & - & - & - & 0 & 5 \\
\hline A5 & 2 & 1 & - & 8 & 1 & - & - & 1 & 7 \\
\hline A6 & - & 1 & 1 & 3 & - & - & - & 0 & 3 \\
\hline A7 & - & - & - & 0 & - & 1 & - & 2 & -2 \\
\hline A8 & - & - & - & 0 & 2 & 1 & - & 4 & -4 \\
\hline A9 & 2 & - & - & 6 & - & - & - & 0 & 6 \\
\hline A10 & - & - & 1 & 1 & - & - & - & 0 & 1 \\
\hline A11 & - & - & - & 0 & - & 1 & 1 & 5 & -5 \\
\hline A12 & - & - & - & 0 & 1 & 2 & - & 5 & -5 \\
\hline
\end{tabular}


Tabla 7. Matriz de relaciones entre pares del método de Copeland.

\begin{tabular}{|c|c|c|c|c|c|c|c|c|c|c|c|c|c|}
\hline & A1 & A2 & $\mathbf{A 3}$ & A4 & A5 & A6 & A7 & $\mathbf{A 8}$ & A9 & A10 & A11 & A12 & \\
\hline A1 & & 0 & 0 & 0 & 0 & 0 & 0 & 0 & 0 & 0 & 0 & 0 & A1 \\
\hline A2 & 1 & & 1 & 0 & 1 & 1 & 1 & 1 & 1 & 1 & 1 & 1 & A2 \\
\hline A3 & 1 & 0 & & 0 & 0 & 0 & 1 & 1 & 0 & 1 & 1 & 1 & A3 \\
\hline A4 & 1 & 1 & 1 & & 0 & 1 & 1 & 1 & 1 & 1 & 1 & 1 & A4 \\
\hline A5 & 1 & 0 & 1 & 1 & & 1 & 1 & 1 & 1 & 1 & 1 & 1 & A5 \\
\hline A6 & 1 & 0 & 1 & 0 & 0 & & 1 & 1 & 0 & 1 & 1 & 1 & A6 \\
\hline A7 & 1 & 0 & 0 & 0 & 0 & 0 & & 1 & 0 & 0 & 1 & 1 & A7 \\
\hline A8 & 1 & 0 & 0 & 0 & 0 & 0 & 0 & & 0 & 0 & 0 & 0 & A8 \\
\hline A9 & 1 & 0 & 1 & 0 & 0 & 1 & 1 & 1 & 0 & 1 & 1 & 1 & A9 \\
\hline A10 & 1 & 0 & 0 & 0 & 0 & 0 & 1 & 1 & 0 & 0 & 1 & 1 & A10 \\
\hline A11 & 1 & 0 & 0 & 0 & 0 & 0 & 0 & 1 & 0 & 0 & & 1 & A11 \\
\hline A12 & 1 & 0 & 0 & 0 & 0 & 0 & 0 & 1 & 0 & 0 & 0 & & A12 \\
\hline & A1 & A2 & A3 & A4 & A5 & A6 & A7 & A8 & A9 & A10 & A11 & A12 & \\
\hline
\end{tabular}

Tabla 8. Resultado de la aplicación del método de Copeland.

\begin{tabular}{|c|c|}
\hline Alternativa & Puntuación \\
\hline A4 & 9 \\
\hline A5 & 9 \\
\hline A2 & 9 \\
\hline A9 & 5 \\
\hline A6 & 3 \\
\hline A3 & 1 \\
\hline A10 & -1 \\
\hline A7 & -3 \\
\hline A11 & -5 \\
\hline A12 & -7 \\
\hline A8 & -9 \\
\hline A1 & -11 \\
\hline
\end{tabular}

Las alternativas A2, A4 y A5 son indiferentes, es decir, son igualmente atractivas para los tomadores de decisión. No hay ninguna manera de escoger una entre esas tres alternativas usando el Método de Copeland. La probabilidad de que un empate ocurra es menor cuanto mayor es el número de tomadores de decisión. Para desempatar, podría incrementarse el número de tomadores de decisión, o podría utilizarse un método cardinal o, incluso, un sorteo entre las tres alternativas indiferentes. Este resultado es diferente al resultado del método del Veto, que sí que escogía una alternativa, la A5.

No obstante, para presentar con más claridad la dependencia de las alternativas irrelevantes del método del Veto, se planteó otra matriz de alternativas ordenadas por cada tomador de decisión (Tabla 9).
Tabla 9. Nueva matriz de alternativas ordenadas por cada tomador de decisión.

\begin{tabular}{|c|c|c|c|c|c|}
\hline Ranking & TD1 & TD2 & TD3 & TD4 & TD5 \\
\hline $\mathbf{1}^{\mathbf{0}}$ & B5 & B4 & B5 & B9 & B9 \\
\hline $\mathbf{2}^{\mathbf{0}}$ & B2 & B5 & B4 & B6 & B4 \\
\hline $\mathbf{3}^{\mathbf{o}}$ & B10 & B6 & B3 & B2 & B3 \\
\hline $\mathbf{4}^{\mathbf{0}}$ & B4 & B2 & B9 & B4 & B6 \\
\hline $\mathbf{5}^{\mathbf{0}}$ & B6 & B9 & B10 & B3 & B12 \\
\hline $\mathbf{6}^{\mathbf{0}}$ & B3 & B10 & B2 & B11 & B10 \\
\hline $\mathbf{7}^{\mathbf{0}}$ & B9 & B3 & B6 & B7 & B8 \\
\hline $\mathbf{8}^{\mathbf{0}}$ & B7 & B7 & B7 & B8 & B2 \\
\hline $\mathbf{9}^{\mathbf{o}}$ & B11 & B12 & B11 & B5 & B5 \\
\hline $\mathbf{1 0}^{\mathbf{o}}$ & B12 & B8 & B8 & B10 & B1 \\
\hline $\mathbf{1 1}^{\mathbf{o}}$ & B8 & B11 & B12 & B12 & B7 \\
\hline $\mathbf{1 2}^{\mathbf{o}}$ & B1 & B1 & B1 & B1 & B11 \\
\hline
\end{tabular}

Para servir como referencias secundarias para el análisis de la ordenación de las alternativas, se utilizaron otros métodos, además del método de Copeland:

- Método de Borda

- Método de Condorcet

- Variación del método del Veto con $0 \%$ de veto, es decir, sin vetar ninguna alternativa.

- Variación del método del Veto con $16,67 \%$ de veto, es decir, vetando las dos alternativas centrales de la matriz ordenada.

- Variación del método del Veto con 33,33\% de veto, es decir, vetando las cuatro alternativas centrales de la matriz ordenada.

- Variación del método del Veto con $66,67 \%$ de veto, es decir, vetando las ocho alternativas centrales de la matriz ordenada. 
- Variación del método del Veto con 83,33\% de veto, es decir, vetando las 10 alternativas centrales de la matriz ordenada.

- Recuento usado en la Fórmula 1 (variación del método de Borda [9]).

El cálculo y resultados aplicando el método del Veto a la nueva matriz de preferencias se presentan en la Tabla 10. La Tabla 11 muestra la matriz de preferencias de Copeland. La comparación entre todos los métodos estudiados aparece en la Tabla 12.

\section{DISCUSIÓN Y CONCLUSIONES}

En los dos casos estudiados el resultado del método del Veto fue diferente al de Copeland.
Para la primera matriz (caso A), cabe destacar que un empate entre las alternativas A2, A4 y A5 es diferente a la victoria de una de ellas (A5) sobre las otras. El empate fue provocado por un ciclo de intransitividad que impide la ordenación justa siendo, por lo tanto, las tres alternativas iguales para ese grupo de tomadores de decisión. En el segundo caso (caso B), la matriz no presenta ningún ciclo de intransitividad, es decir, el resultado del método de Copeland es independiente de las alternativas irrelevantes para esa matriz de preferencias. Como el resultado del método del Veto (B5) es diferente al del método de Copeland (B4), queda probado que el método del Veto es dependiente de las alternativas irrelevantes.

Tabla 10. Resultados parciales y final aplicando el método del Veto a la matriz nueva.

\begin{tabular}{|c|c|c|c|c|c|c|c|c|c|}
\hline \multirow{2}{*}{ Alternativa } & \multicolumn{4}{|c|}{ FUERZA } & \multicolumn{4}{|c|}{ DEBILIDAD } & \multirow{2}{*}{$\alpha$} \\
\hline & $1^{\mathrm{a}}$ & $2^{\mathrm{a}}$ & $3^{\mathrm{a}}$ & Subtotal & $10^{\mathrm{a}}$ & $11^{\mathrm{a}}$ & $12^{\mathrm{a}}$ & Subtotal & \\
\hline B1 & - & - & - & 0 & 1 & - & 4 & -13 & -13 \\
\hline B2 & - & 1 & 1 & 3 & - & - & - & 0 & 3 \\
\hline B3 & - & - & 2 & 2 & - & - & - & 0 & 2 \\
\hline B4 & 1 & 2 & - & 7 & - & - & - & 0 & 7 \\
\hline B5 & 2 & 1 & - & 8 & - & - & - & 0 & 8 \\
\hline B6 & - & 1 & 1 & 3 & - & - & - & 0 & 3 \\
\hline B7 & - & - & - & 0 & - & 1 & - & -2 & -2 \\
\hline B8 & - & - & - & 0 & 2 & 1 & - & -4 & -4 \\
\hline B9 & 2 & - & - & 6 & - & - & - & 0 & 6 \\
\hline B10 & - & - & 1 & 1 & 1 & - & - & -1 & $\mathbf{0}$ \\
\hline B11 & - & - & - & 0 & - & 1 & 1 & -5 & -5 \\
\hline B12 & - & - & - & 0 & 1 & 2 & - & -5 & -5 \\
\hline
\end{tabular}

Tabla 11. Nueva matriz de relaciones entre pares del método de Copeland.

\begin{tabular}{|c|c|c|c|c|c|c|c|c|c|c|c|c|c|}
\hline & B1 & B2 & B3 & B4 & B5 & B6 & B7 & B8 & B9 & B10 & B11 & B12 & \\
\hline B1 & & 0 & 0 & 0 & 0 & 0 & 0 & 0 & 0 & 0 & 0 & 0 & B1 \\
\hline B2 & 1 & & 1 & 0 & 0 & 0 & 1 & 1 & 0 & 1 & 1 & 1 & B2 \\
\hline B3 & 1 & 0 & & 0 & 0 & 0 & 1 & 1 & 0 & 1 & 1 & 1 & B3 \\
\hline B4 & 1 & 1 & 1 & & 1 & 1 & 1 & 1 & 1 & 1 & 1 & 1 & B4 \\
\hline B5 & 1 & 1 & 1 & 0 & & 1 & 1 & 1 & 1 & 1 & 1 & 1 & B5 \\
\hline B6 & 1 & 1 & 1 & 0 & 0 & & 1 & 1 & 0 & 1 & 1 & 1 & B6 \\
\hline B7 & 1 & 0 & 0 & 0 & 0 & 0 & & 1 & 0 & 0 & 1 & 1 & B7 \\
\hline B8 & 1 & 0 & 0 & 0 & 0 & 0 & 0 & & 0 & 0 & 0 & 0 & B8 \\
\hline B9 & 1 & 1 & 1 & 0 & 0 & 1 & 1 & 1 & 0 & 1 & 1 & 1 & B9 \\
\hline B10 & 1 & 0 & 0 & 0 & 0 & 0 & 1 & 1 & 0 & 0 & 1 & 1 & B10 \\
\hline B11 & 1 & 0 & 0 & 0 & 0 & 0 & 0 & 1 & 0 & 0 & & 1 & B11 \\
\hline B12 & 1 & 0 & 0 & 0 & 0 & 0 & 0 & 1 & 0 & 0 & 0 & & B12 \\
\hline & B1 & B2 & B3 & B4 & B5 & B6 & B7 & B8 & B9 & B10 & B11 & B12 & \\
\hline
\end{tabular}


Tabla 12. Comparativa de resultados con los diversos métodos estudiados para la nueva matriz de preferencias.

\begin{tabular}{|c|c|c|c|c|c|c|c|c|c|c|}
\hline & PAPER & COPELAND & BORDA & CONDORCET & $\begin{array}{c}\text { VETO } \\
\mathbf{0 \%}\end{array}$ & $\begin{array}{c}\text { VETO } \\
\mathbf{1 6 , 7 \%}\end{array}$ & $\begin{array}{c}\text { VETO } \\
\mathbf{3 3 , 3} \%\end{array}$ & $\begin{array}{c}\text { VETO } \\
\mathbf{6 6 , 7 \%}\end{array}$ & $\begin{array}{c}\text { VETO } \\
\mathbf{8 3 , 3 \%}\end{array}$ & F1 \\
\hline B1 & 12 & 12 & 12 & 12 & 12 & 12 & 12 & 12 & 12 & 12 \\
\hline B2 & 4 & 5 & 5 & 5 & 4 & 5 & 4 & 4 & 4 & 5 \\
\hline B3 & 6 & 6 & 6 & 6 & 6 & 6 & 6 & 6 & 4 & 6 \\
\hline B4 & 2 & 1 & 1 & 1 & 1 & 1 & 1 & 2 & 3 & 1 \\
\hline B5 & 1 & 2 & 4 & 2 & 4 & 3 & 2 & 1 & 1 & 3 \\
\hline B6 & 4 & 4 & 3 & 4 & 3 & 3 & 4 & 4 & 4 & 4 \\
\hline B7 & 8 & 8 & 8 & 8 & 8 & 8 & 8 & 8 & 4 & 8 \\
\hline B8 & 9 & 11 & 9 & 11 & 10 & 9 & 9 & 8 & 4 & 10 \\
\hline B9 & 3 & 3 & 2 & 3 & 2 & 2 & 2 & 2 & 1 & 2 \\
\hline B10 & 7 & 7 & 7 & 7 & 7 & 7 & 7 & 6 & 4 & 7 \\
\hline B11 & 10 & 9 & 11 & 9 & 10 & 11 & 10 & 11 & 11 & 10 \\
\hline B12 & 10 & 10 & 9 & 10 & 9 & 10 & 10 & 10 & 4 & 9 \\
\hline
\end{tabular}

Este hecho era ya sabido por los autores; no obstante, en el epígrafe 5 del artículo [1], sobre la discusión de los resultados, cuando analizan el axioma de Arrow sobre dependencia de las alternativas irrelevantes, los autores dicen A pesar de que el método propuesto es sensible a la independencia de las alternativas irrelevantes - una de las propiedades del Teorema de Arrow, ésta ha sido reducida mediante la limitación de las alternativas en los cuartiles superior e inferior [...]. De este modo, el análisis se concentra dentro del $50 \%$ de las alternativas relevantes. Esta afirmación es incorrecta.

En el segundo caso, el método de Borda, que es dependiente de las alternativas irrelevantes, tiene el mismo resultado que el método de Condorcet y el método de Copeland. Por tanto, puede decirse con propiedad que el método del Veto no puede ser considerado mejor que el recuento Borda.

Por otro lado, el método de Copeland es preferible al Recuento de Borda; así, el método de Copeland puede ser considerado preferible al método del Veto.

El propio método del Veto, cuando se modifica el porcentaje de alternativas vetadas, varía su resultado. Cuando el veto fue igual o inferior a cuatro de las 12 alternativas, el resultado fue el mismo que para Copeland (B4). Pero cuando el veto fue igual o superior a seis alternativas, el resultado fue B5 y
B5 empatado con B9. Esto significa que el veto de los dos cuartiles intermedios puede estar retirando información relevante para obtener un resultado más justo.

Otra conclusión importante es el hecho de que alternativas consideradas muy desfavorables influyen notablemente en el resultado. Así, en caso de que algunas alternativas sean ordenadas en las últimas posiciones por la mayoría de los tomadores de decisión, estas van a alterar el resultado final, ya que alternativas que puedan ser favorables para unos tomadores de decisión pero no para otros van a perder debilidad, con lo que la fuerza va a influir más sobre el índice $\alpha$. Esto permite fácilmente la manipulación de los resultados a través de la selección de las alternativas, lo que denota alta sensibilidad a las alternativas irrelevantes. Esta característica puede observarse en el Ejemplo 1, que compara el método de Copeland con el método del Veto para un grupo de alternativas en el cual una de ellas es muy desfavorable. La Tabla 13 presenta los datos iniciales del Ejemplo 1. La Tabla 14 muestra el veto para la matriz original del Ejemplo 1. La Tabla 15 representa la nueva matriz de decisión después de quitar la alternativa muy desfavorable y el veto aplicado sobre ella.

Ejemplo 1 - Comparación entre el método del Veto y el método de Copeland con una alternativa muy desfavorable. 
Tabla 13. Matriz de decisión.

\begin{tabular}{|c|c|c|c|c|c|}
\hline Ranking & TD1 & TD2 & TD3 & TD4 & TD5 \\
\hline $1^{\text {' }}$ & $\mathrm{Z} 1$ & $\mathrm{Z} 2$ & $\mathrm{Z} 2$ & $\mathrm{Z} 4$ & $\mathrm{Z} 4$ \\
\hline $2^{\text {o }}$ & $\mathrm{Z} 3$ & $\mathrm{Z} 3$ & $\mathrm{Z} 3$ & $\mathrm{Z} 3$ & $\mathrm{Z} 3$ \\
\hline $3^{\text {o }}$ & $\mathrm{Z} 4$ & $\mathrm{Z} 4$ & $\mathrm{Z} 1$ & $\mathrm{Z} 1$ & $\mathrm{Z} 2$ \\
\hline $4^{\mathbf{o}}$ & $\mathrm{Z} 2$ & $\mathrm{Z} 1$ & $\mathrm{Z} 4$ & $\mathrm{Z} 2$ & $\mathrm{Z} 1$ \\
\hline $5^{\circ}$ & $\mathrm{Z} 5$ & $\mathrm{Z} 5$ & $\mathrm{Z} 5$ & $\mathrm{Z} 5$ & $\mathrm{Z} 5$ \\
\hline
\end{tabular}

Tabla 14. Matriz con Veto.

\begin{tabular}{|c|c|c|c|c|c|}
\hline Ranking & TD1 & TD2 & TD3 & TD4 & TD5 \\
\hline $1^{\text {er }}$ cuartil & Z1 & Z2 & Z2 & Z4 & Z4 \\
\hline Veto & Z3 & Z3 & Z3 & Z3 & Z3 \\
\hline Veto & Z4 & Z4 & Z1 & Z1 & Z2 \\
\hline Veto & Z2 & Z1 & Z4 & Z2 & Z1 \\
\hline $4^{\text {o }}$ cuartil & Z5 & Z5 & Z5 & Z5 & Z5 \\
\hline
\end{tabular}

Para esta matriz de decisión la alternativa vencedora es Z2 empatada con Z4. La ordenación de las alternativas resultante fue Z2 = Z4, Z1, Z3, Z5. No obstante, la alternativa $Z 5$ es claramente desfavorable para todos los tomadores de decisión. Por tanto, se retiró esa alternativa para calcular de nuevo el resultado.

Tabla 15. Matriz de decisión y veto después de eliminar la alternativa Z5.

\begin{tabular}{|c|c|c|c|c|c|}
\hline Ranking & TD1 & TD2 & TD3 & TD4 & TD5 \\
\hline $1^{\text {er }}$ cuartil & Z1 & Z2 & Z2 & Z4 & Z4 \\
\hline Veto & Z3 & Z3 & Z3 & Z3 & Z3 \\
\hline Veto & Z4 & Z4 & Z1 & Z1 & Z2 \\
\hline $4^{\text {c }}$ cuartil & Z2 & Z1 & Z4 & Z2 & Z1 \\
\hline
\end{tabular}

Para esta nueva matriz de decisión la alternativa vencedora fue $Z 4$. La ordenación de las alternativas fue Z4, Z2, Z3, Z1.

Aplicando para este ejemplo el método de Copeland, tanto en la matriz con las cinco alternativas, que incluye Z5, irrelevante, como la que no la incluye, el resultado del cálculo es el mismo: Z3. En la primera matriz, la ordenación resultante fue $Z 3$, Z4, Z2, Z1, Z5. En la segunda matriz fue Z3, Z4, $\mathrm{Z} 2$, Z1. Como no existen ciclos de intransitividad, el resultado es independiente de las alternativas irrelevantes.

En este ejemplo se observa claramente que la alternativa Z5 no debe estar entre las alternativas consideradas, pero eso no es óbice para que el método de Copeland realice el cálculo correctamente, mientras que el método del Veto denota alta dependencia de la alternativa irrelevante o, si se prefiere para este caso, de la alternativa altamente desfavorable. Además, el método del Veto coloca a la alternativa escogida por Copeland (Z3) en la penúltima posición en las dos matrices (cuarta y tercera colocación).

Esto refuerza el concepto de que el Veto no reduce la dependencia de las alternativas irrelevantes, sino que puede retirar información esencial para una ordenación justa, como en el Ejemplo 1. También reforzando lo que ya ha sido expuesto, el recuento de Borda fue aplicado a las dos matrices de este ejemplo y el resultado fue la misma ordenación que se obtuvo con el método de Copeland.

En el artículo original, los autores incluyen el denominado coeficiente $\beta$ para retirar las alternativas que no son suficientemente preferibles, es decir, aquellas con las que el grupo tiene un elevado grado de desacuerdo.

Si $f_{i} \geq \beta \cdot F_{i} \rightarrow \mathrm{A}_{i}$ es descartada

Este coeficiente pretende resolver el problema de las alternativas extremadamente débiles, como podría ser el caso de Z5 en el Ejemplo 1, pero su aplicación podría complicar mucho la utilización de este método. De hecho, su aplicación parece resultar inviable hasta para los propios autores, que no usan el coeficiente $\beta$ en el artículo.

El valor de este coeficiente debería ser estipulado por los tomadores de decisión. Como no se menciona un posible valor en el artículo, para evaluar y entender el efecto del coeficiente se consideró $\beta=\infty$. La aplicación del concepto del coeficiente $\beta$ se muestra en la Tabla 16, Tabla 17, Tabla 18 y Tabla 19.

Con la matriz de preferencias original el resultado fue A5. Las alternativas que presentan debilidad, pero no fuerza, cumplen que $\beta=\infty$, y fueron retiradas para recalcular las preferencias. Estas fueron A1, A7, A8, A11 y A12.

Así (Tabla 17), el resultado sería la alternativa A2 empatada con A4. No obstante, con la nueva matriz, las alternativas que presentan debilidad, pero no fuerza, fueron A10 y A3.

El resultado en este caso (Tabla 18), sería la alternativa A2. A pesar de aplicar en dos ocasiones 
Tabla 16. Evolución de la matriz de decisión aplicando el coeficiente $\beta$.

\begin{tabular}{|c|c|c|c|c|c|}
\hline Ranking & TD1 & TD2 & TD3 & TD4 & TD5 \\
\hline $\mathbf{1}^{\mathbf{0}}$ & A5 & A2 & A5 & A9 & A9 \\
\hline $\mathbf{2}^{\mathbf{0}}$ & A2 & A5 & A4 & A6 & A4 \\
\hline $\mathbf{3}^{\mathbf{0}}$ & A10 & A6 & A3 & A4 & A3 \\
\hline $4^{\mathbf{0}}$ & A4 & A4 & A2 & A2 & A6 \\
\hline $\mathbf{5}^{\mathbf{0}}$ & A6 & A9 & A10 & A3 & A12 \\
\hline $\mathbf{6}^{\mathbf{0}}$ & A3 & A10 & A9 & A11 & A10 \\
\hline $\mathbf{7}^{\mathbf{0}}$ & A9 & A3 & A6 & A7 & A8 \\
\hline $\mathbf{8}^{\mathbf{o}}$ & A7 & A7 & A7 & A8 & A2 \\
\hline $\mathbf{9}^{\mathbf{0}}$ & A11 & A12 & A11 & A10 & A5 \\
\hline $\mathbf{1 0}^{\mathbf{0}}$ & A12 & A8 & A8 & A5 & A1 \\
\hline $\mathbf{1 1}^{\mathbf{0}}$ & A8 & A14 & A12 & A12 & A7 \\
\hline $\mathbf{1 2}^{\mathbf{0}}$ & A4 & A4 & A4 & A1 & A14 \\
\hline
\end{tabular}

Tabla 17. Nueva matriz de decisión después de retirar de la original las alternativas con debilidad extrema.

\begin{tabular}{|c|c|c|c|c|c|}
\hline Ranking & TD1 & TD2 & TD3 & TD4 & TD5 \\
\hline $\mathbf{1}^{\mathbf{0}}$ & A5 & A2 & A5 & A9 & A9 \\
\hline $\mathbf{2}^{\mathbf{0}}$ & A2 & A5 & A4 & A6 & A4 \\
\hline $\mathbf{3}^{\mathbf{0}}$ & A10 & A6 & A3 & A4 & A3 \\
\hline $\mathbf{4}^{\mathbf{0}}$ & A4 & A4 & A2 & A2 & A6 \\
\hline $\mathbf{5}^{\mathbf{0}}$ & A6 & A9 & A10 & A3 & A10 \\
\hline $\mathbf{6}^{\mathbf{0}}$ & A3 & A10 & A9 & A10 & A2 \\
\hline $\mathbf{7}^{\mathbf{0}}$ & A9 & A3 & A6 & A5 & A5 \\
\hline
\end{tabular}

Tabla 18. Matriz después de retirar las alternativas A10 y A3.

\begin{tabular}{|c|c|c|c|c|c|}
\hline Ranking & TD1 & TD2 & TD3 & TD4 & TD5 \\
\hline $\mathbf{1}^{\mathbf{0}}$ & A5 & A2 & A5 & A9 & A9 \\
\hline $\mathbf{2}^{\mathbf{0}}$ & A2 & A5 & A4 & A6 & A4 \\
\hline $\mathbf{3}^{\mathbf{0}}$ & A4 & A6 & A2 & A4 & A6 \\
\hline $\mathbf{4}^{\mathbf{0}}$ & A6 & A4 & A9 & A2 & A2 \\
\hline $\mathbf{5}^{\mathbf{0}}$ & A9 & A9 & A6 & A5 & A5 \\
\hline
\end{tabular}

Tabla 19. Matriz consistente de acuerdo al coeficiente $\beta$, después de retirar la alternativa A6.

\begin{tabular}{|c|c|c|c|c|c|}
\hline Ranking & TD1 & TD2 & TD3 & TD4 & TD5 \\
\hline $\mathbf{1}^{\mathbf{0}}$ & A5 & A2 & A5 & A9 & A9 \\
\hline $\mathbf{2}^{\mathbf{0}}$ & A2 & A5 & A4 & A4 & A4 \\
\hline $\mathbf{3}^{\mathbf{0}}$ & A4 & A4 & A2 & A2 & A2 \\
\hline $\mathbf{4}^{\mathbf{0}}$ & A9 & A9 & A9 & A5 & A5 \\
\hline
\end{tabular}

el coeficiente $\beta$, la tercera matriz todavía presenta una alternativa con debilidad extrema, la A6.

El resultado después de aplicar el coeficiente $\beta$ (Tabla 19) fue un empate entre las alternativas A5 y la A9. Ese resultado es diferente al presentado en el artículo, que dejaba como única solución A5, mientras que A9 quedaba en segunda posición, pero también diferente al resultado del método de Copeland, para el que había un empate entre A2, A4 y A5.

Además se observa que se anularon 8 de 12 alternativas (A1, A7, A8, A11, A12, A10, A3, A6), es decir, $66,7 \%$ del total de las alternativas fueron extremadamente débiles de acuerdo con el coeficiente $\beta$. Este hecho debería ser suficiente para que el consultor que escogió las alternativas que serían votadas se plantease si la selección había sido correcta.

Las alternativas extremadamente débiles sirvieron para modificar el resultado, dejando clara la dependencia que el método del Veto tiene de las alternativas irrelevantes.

Otra consideración es que el método del Veto fue creado para atender a la toma de decisiones en materia de recursos hídricos. No obstante, el hecho de elegir solo una alternativa debilita la acción de este método en ese campo, ya que no considera el valor de la inversión necesaria en cada alternativa. Es decir, una alternativa puede tener un coste de implantación equivalente al de diversas alternativas juntas. Ese parámetro debe ser considerado en este tipo de decisiones, ya que es esencial para gestionar el presupuesto de cualquier organización.

\section{AGRADECIMIENTOS}

$\mathrm{Al}$ Conselho Nacional de Desenvolvimento Científico e Tecnológico (CNPq) por su apoyo financiero.

\section{REFERENCIAS}

[1] D.C. Morais and A.T. de Almeida. "Group decision making on water resources based on analysis of individual rankings". Omega. Vol. 40, Issue 1, pp. 42-52. January, 2012. DOI:10.1016/j.omega.2011.03.005. 
[2] A. Candia-Véjar y M. González. "Sistemas de ingeniería: Problemas, modelos y algoritmos de solución para la ayuda en la toma de decisiones". Ingeniare. Revista chilena de ingeniería. Vol. $19 \mathrm{~N}^{\circ}$ 3, pp. 310-311. 2011. ISSN: 0718-3305.

[3] E. Forman and K. Peniwati. "Aggregating individual judgments and priorities with the analytic hierarchy process". European Journal of Operational Research. Vol. 108, Issue 1, pp. 165-169. July, 1998. DOI: 10.1016/S0377-2217(97)00244-0.

[4] L.F.A.M. Gomes, M.C. González Araya e C. Carignano. "Tomada de decisões em cenários complexos". Thomson. São Paulo, Brasil. ISBN: 9788522103546. 2004.

[5] S. Barba-Romero y J.-C. Pomerol. "Decisiones multicriterio: Fundamentos teóricos y utilización práctica". Universidad de Alcalá de Henares. Vol. 4. Alcalá de Henares, España. ISBN: 9788481381801.1997.

[6] K.J. Arrow. "Social choice and individual values". John Wiley \& Sons, Inc. New York, USA. 1951.

[7] K.J. Arrow. "A difficulty in the concept of social welfare". Journal of Political Economy. Vol. 58, Issue 4, pp. 328-346. August, 1950.

[8] J.E.D. Mattos Fernandes, L.F.A. Monteiro Gomes, J.C.C.B. Soares de Mello e S.F. Gomes Júnior. "Seleção de uma aeronave para atividades de charter regional com utilização do método multicritério de Borda modificado com utilização de mediana". Journal of Transport Literature. Vol. $7 \mathrm{~N}^{\circ} 2$. 2012. ISSN: 2238-1031.

[9] J.C. Correia Baptista Soares de Mello, L.F. Gomes Autran Monteiro, E. Gonçalves Gomes and M.H. Campos Soares de Mello. "Use of ordinal multi-criteria methods in the analysis of the Formula 1 World Championship". Cadernos EBAPE.BR. Vol. 3, Issue 2, pp. 01-08. July, 2005.

[10] G. Hägele and F. Pukelsheim. "Llull's writings on electoral systems". Studia Lulliana. Vol. 41, Issue 97, pp. 3-38. 2001.

[11] J.-C. Borda, "Mémoire sur les élections au scrutin: Histoire de l'Académie Royale des Sciences”. Paris, France. 1781.
[12] E. Gonçalves Gomes, J.C.C.B. Soares de Mello e J.A.D.C. Mangabeira. "Avaliação de desempenho de agricultores familiares com o método multicritério de Copeland". Pesquisa Operacional para o Desenvolvimento. Vol. 1 $\mathrm{N}^{\circ}$ 2, pp. 159-168. 2009.

[13] J.-A.-N. de Caritat, Marquis de Condorcet. "Essai sur l'application de l'analyse a la probabilité des décisions rendues a la pluralité des voix". Imprimerie Royale. Paris. 1785.

[14] M.H. Campos Soares de Mello, H.L.M. de Meirelles Quintella e J.C. Correia Baptista Soares de Mello. "Avaliação do desempenho de alunos considerando classificações obtidas e opiniões dos docentes". Investigação Operacional. Vol. $24 \mathrm{~N}^{\circ}$ 2, pp. 187-196. Diciembre 2004. ISSN: 0874-5161.

[15] A.H. Copeland. "A 'reasonable' social welfare function. Seminar on applications of mathematics to social sciences". University of Michigan Seminar on Applications of Mathematics to the Social Sciences. 1951.

[16] S.F. Gomes Júnior, J.C. Correia Baptista Soares de Mello e M.H.Campos Soares de Mello. "Utilização do método de Copeland para avaliação dos polos regionais do CEDERJ". Rio's International Journal on Sciences of Industrial and Systems Engineering and Management. Vol. 2, Issue 1, pp. 87-98. 2008.

[17] J.C. Pomerol and S. Barba-Romero. "Multicriterion decision in management: Principles and practice". Kluwer Academic. Boston, Massachusetts, Estados Unidos. 2000.

[18] J.C.C.B. Soares de Mello, E.G. Gomes, M.P.E. Lins e A.J.R. Soares de Mello. "Uso da Pesquisa Operacional em esportes: o caso das Olimpíadas". Boletim da Sociedade Brasileira de Pesquisa Operacional. Vol. 19, pp. 5-6. 2001.

[19] S.F. Gomes Júnior, J.C.C.B. Soares de Mello and L.A. Meza. "Sequential use of ordinal multicriteria methods to obtain a ranking for the 2012 Summer Olympic Games". WSEAS Transaction on Systems. Vol. 13. 2014. 